\title{
Boundary layer flow of mixed convection viscoelastic micropolar fluid over a horizontal circular cylinder with aligned magnetohydro dynamic effect
}

\author{
Laila Amera Aziz a, ${ }^{\star}$, Abdul Rahman Mohd Kasim ${ }^{\text {a, }}{ }^{,}$, Mohd Zuki Salleh a, Sharidan Shafie ${ }^{b}$, Nur \\ Syahidah Yusoff a \\ a Applied \& Industrial Mathematics Research Group, Faculty of Science \& Industrial Technology, Universiti Malaysia Pahang, 26300 Kuantan, \\ Pahang, Malaysia \\ ${ }^{b}$ Deparment of Mathematical Sciences., Faculty of Science, Universiti Teknologi Malaysia, 81310 Johor, Malaysia \\ *Corresponding author: rahmanmohd@ump.edu.my / laila@ump.edu.my
}

\section{Article history}

Received 18 February 2017

Accepted 1 October 2017

\begin{abstract}
The boundary layer flow of a viscoelastic micropolar fluid over a horizontal circular cylinder with aligned magnetohydrodynamic effect is considered. The governing boundary layer equations are transformed into non-dimensional form by using appropriate dimensionless variables. The nondimensional governing equations are then transformed into similarity equations and solved using an implicit finite difference scheme known as the Keller box method. Numerical results on the distributions of velocity and temperature of fluid are obtained for a range of values of magnetic parameter, $M$, viscoelastic parameter, $K$, material parameter, $K_{1}$, and mixed convection parameter, $\lambda$. The graphical representation of the results are presented and it shows that the investigated parameters are significance and affected the fluid flow.
\end{abstract}

Keywords: Viscoelastic micropolar, horizontal circular cylinder, mixed convection, aligned MHD

\section{INTRODUCTION}

Convection is one of the heat transfer method that can be categorised as natural convection, forced convection, as well as the combination of both known as the mixed convection. Convection is a very important process with practical importance in industrial, geology and even in our daily life. Most electrical appliances such as electric kettle, refrigerator and air-conditioner functioned based on convection process which enable the transfer of heat from one place to another by fluid movement.

The fluid of interest in this study is the non-Newtonian viscoelastic micropolar fluid. The study of convective boundary layer flow of micropolar and viscoelastic fluid over various geometrical shapes are well known among researchers and both type of fluids are equally important in various fields. Viscoelastic fluid is a fluid which displays the properties of being both viscous and elastic such as tooth paste, unset cement and waxy crude oil. On the other hand, micropolar fluid is a fluid with microstructure consisting of dumb-bell molecules or short rigid cylindrical element. Such fluid includes liquid crystals, animal bloods and lubricants.

Among the pioneer of viscoelastic fluid modelling is (Rajagopal et al., 1984) who studied the boundary layer flow of viscoelastic fluid over stretching sheet which is highly relevent in polymer industry. The idea is then extended by (Dandapat and Gupta, 1989), who considers the heat transfer of that flow over a stretching sheet. Since then, the study of viscoelastic study over various geometrical shapes gained popularity among researchers due to numerous industrial practicality.

The study of viscoelastic fluid over circular cylinder is significant in oil and gas well production as it can contribute to drag reduction (Xiong et al., 2010). The findings from such study is beneficial to reduce friction, wear, erosion, corrosion, and deposits for well construction, completion and production of oil and gas (Jin et al., 2013). Among the researches on viscoelastic fluid over a circular cylinder is the published articles by (Anwar et al., 2008) who studied the mixed convection boundary layer flow of both cases of heated and cooled cylinders, (Kasim et al., 2011) investigates the free convection boundary layer flow with the presence of heat generation while (Huang and Feng, 1995) considers the wall effect on the flow of viscoelastic fluid.

The study of flow of micropolar fluid is also on demand due to various industrial and engineering applications such as thrust bearing technologies, solidification of liquid crystal as well as colloid and polymeric suspensions. Numerous studies including (Naduvinamani and Kadadi, 2013), (Allen and Kline, 1971), (Das et al., 2004) and (Rahmatabadi et al., 2010)) have demonstrated that micropolar fluid is notably useful as lubricants for bearing systems as it increases load carrying capacity and lower the coefficient of friction as opposed to when system is lubricated by Newtonian fluids.

The first model of micropolar fluid is introduced by (Eringen, 1964) and (Eringen, 1965). A more recent study by (Eringen, 2001) claims that the micropolar fluid model is well-matched with certain 
biological fluids, magnetic fluids, liquid with anisotropic property such as liquid crystal, muddy fluids as well as dusty clouds.

In recent years, there has been an increasing amount of literature on micropolar fluid since Eringen's first discovery. To date, numerous studies have attempted to describe the blood flow model as micropolar fluid (refer (Ellahi et al., 2014), (Misra et al., 2014), (Mekheimer et al., 2016)), thus convincing the authors the importance of micropolar fluid for medical sciences break through. While some researchers decided on practical approach, a number of authors instigated a numerical approach of their proposed micropolar model by solving the related equations and explaining the changes of flow and heat transfer of the fluid from the parameters involved in the equations. (Qasim et al., 2013), for instance, solved the heat transfer problem of micropolar fluid over stretching sheet using the method of RungeKutta-Fehlberg fourth-fifth order while (Nazar et al., 2004), (Ishak et al., 2007), (Salleh et al., 2009) and (Alkasasbeh et al., 2015) conducted their analyses using finite difference method known as the Keller-box method.

However, in this study, the non-Newtonian viscoelastic and micropolar fluid will not be considered individually as prior studies, but together as a whole. To the best of authors' knowledge, the study of convective boundary layer flow and heat transfer of viscoelastic micropolar fluid has not yet been addressed. This study is motivated from the existence of certain fluids that could fit both characteristics as being viscoelastic as well as micropolar fluid, for example human and animal blood. With the existence of viscoelastic micropolar model, a more accurate model of these types of fluid can be used to explain the flow and heat transfer of such fluid and contribute to various fields as was mentioned above.

This investigation will also consider the presence of magnetic field. Magnetic field has wide range of applications including to determine the mass flow rate of petroleum through pipelines. Although there are diverse selections of flow meters to choose from, the electromagnetic flow meters is preeminent as it is robust and provide the same measurement in laminar and turbulent flow (Ustinov, 2016). Hence, in this study we attempted to investigate the influence of multiple parameters such as viscoelastic, micro rotation and magnetic parameter on the boundary layer flow and heat transfer of viscoelastic micropolar fluid as it passes a circular cylinder.

\section{MATHEMATICAL FORMULATION}

Consider a horizontal circular cylinder with radius $a$ heated with a costant temperature, $T_{w}$ and immersed in a viscoelastic micropolar fluid. A uniform free stream $\left(1 / 2 U_{\infty}\right)$ is flowing upward so that according to (Merkin, 1977), the free stream velocity $\bar{u}_{e}(\bar{x})$ for the boundary layer equation is $\bar{u}_{e}(\bar{x})=U_{\infty} \sin (\bar{x} / a)$ and the ambient temperature is $T_{\infty}$ as shown in the following figure.

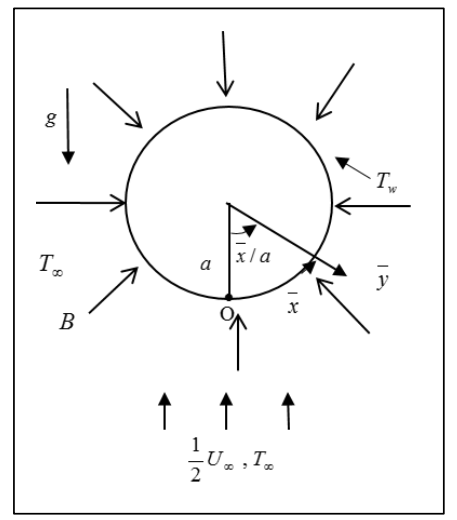

Fig. 1 Physical model and coordinate system for mixed convection of a horizontal circular cylinder.

Under the Boussinesq and boundary-layer approximations, according to (Mohd Kasim et al., 2013) and (Nazar et al., 2003), the basic non- dimensionalised Navier-Stokes equations describing the flow are given by:

Continuity equation:

$\frac{\partial \bar{u}}{\partial \bar{x}}+\frac{\partial \bar{v}}{\partial \bar{y}}=0$

Momentum equation:

$\bar{u} \frac{\partial \bar{u}}{\partial \bar{x}}+\bar{v} \frac{\partial \bar{u}}{\partial \bar{y}}=\bar{u}_{e} \frac{\partial \bar{u}_{e}}{\partial \bar{x}}+\left(\frac{\mu+\kappa}{\rho}\right) \frac{\partial^{2} \bar{u}}{\partial \bar{y}^{2}}+\frac{k_{0}}{\rho}\left[\frac{\partial}{\partial \bar{x}}\left(\bar{u} \frac{\partial^{2} \bar{u}}{\partial \bar{y}^{2}}\right)+\bar{v} \frac{\partial^{3} \bar{u}}{\partial \bar{y}^{3}}-\frac{\partial \bar{u}}{\partial \bar{y}} \frac{\partial^{2} \bar{u}}{\partial \bar{x} \partial \bar{y}}\right]$

$+g \beta\left(T-T_{\infty}\right) \sin \left(\frac{\bar{x}}{a}\right)+\frac{\kappa}{\rho} \frac{\partial \bar{H}}{\partial \bar{y}}-\frac{\sigma}{\rho}\left(\bar{u}-\bar{u}_{e}\right) B^{2} \sin ^{2} \alpha$

Energy equation:

$\bar{u} \frac{\partial T}{\partial \bar{x}}+\bar{v} \frac{\partial T}{\partial \bar{y}}=\alpha \frac{\partial^{2} T}{\partial \bar{y}^{2}}$

Micropolar equation:

$\rho j\left(\bar{u} \frac{\partial \bar{H}}{\partial \bar{x}}+\bar{v} \frac{\partial \bar{H}}{\partial \bar{y}}\right)=-\kappa\left(2 \bar{H}+\frac{\partial \bar{u}}{\partial \bar{y}}\right)+\gamma \frac{\partial^{2} \bar{H}}{\partial \bar{y}^{2}}$

subject to the boundary conditions:

$\bar{u}=\bar{v}=0, \quad T=T_{w}, \quad \bar{H}=-n \frac{\partial \bar{u}}{\partial \bar{y}} \quad$ on $\quad \bar{y}=0$,

$\bar{u}=\bar{u}_{e}(x), \quad \frac{\partial \bar{u}}{\partial \bar{y}}=0, \quad T=T_{\infty}, \quad \bar{H}=0 \quad$ as $\bar{y} \rightarrow \infty$

where $\bar{u}$ and $\bar{v}$ are velocity components along the $x$ and $y$ directions of the cylinder surface, $\bar{u}_{e}$ is the velocity outside the boudary layer, $v$ is the kinematic viscosity, $k_{0}(\geq 0)$ is the viscoelasticity parameter, $\rho$ and $T$ is the fluid desity and temperature, respectively, $\bar{H}$ is the total spin or microrotation component, $\sigma$ is the electrical conductivity, $\alpha$ is the thermal diffusivity of fluid and $j$ is the microinertia density defined as $j=\frac{a v}{U_{\infty}}$. $\quad \gamma$, on the other hand is a constant given by $\gamma=\left(\mu+\frac{\kappa}{2}\right) j$

Then, appropriate combinations of parameters and constants of the equations and flow characteristics known as dimensionless variables are proposed to trasform Eq. (1)-(5) to dimensionless equations. As a result of the non-dimensionalisation process, the complexity of the equations and the number of parameters are reduced.

$$
\begin{aligned}
& x=\frac{\bar{x}}{a}, \quad y=\operatorname{Re}^{1 / 2}\left(\frac{\bar{y}}{a}\right), \quad u=\frac{\bar{u}}{U_{\infty}}, \quad v=\operatorname{Re}^{1 / 2}\left(\frac{\bar{v}}{U_{\infty}}\right) \\
& H=\operatorname{Re}^{-1 / 2}\left(\frac{a}{U_{\infty}}\right) \bar{H}, \quad u_{e}=\frac{\bar{u}_{e}(\bar{x})}{U_{\infty}}, \quad \theta=\frac{T-T_{\infty}}{T_{w}-T_{\infty}}
\end{aligned}
$$

where $\operatorname{Re}$ represents the Reynolds number defined by $\operatorname{Re}=\frac{U_{\infty} a}{v}$.

Reynolds number is a dimensionless number which represents the ratio of bouyancy force and viscous force acting on the fluid for forced convection cases. The same role is played by Grashof number that will be introduced later, in natural convection. As the problem considered is mixed convection, both dimensionless numbers are present in our equations.

Substituting Eq. (6) into Eq. (1)-(4), the following dimensionless equations are produced.

Continuity equation:

$\frac{\partial u}{\partial x}+\frac{\partial v}{\partial y}=0$

Momentum equation: 


$$
\begin{aligned}
u \frac{\partial u}{\partial x}+v \frac{\partial u}{\partial y}= & u_{e} \frac{\partial u_{e}}{\partial x}+\left(1+K_{1}\right) \frac{\partial^{2} u}{\partial y^{2}}+K\left(\frac{\partial}{\partial x}\left(u \frac{\partial^{2} u}{\partial y^{2}}\right)+v \frac{\partial^{3} u}{\partial y^{3}}-\frac{\partial u}{\partial y} \frac{\partial^{2} u}{\partial x \partial y}\right) \\
& +\lambda \theta \sin x+K_{1} \frac{\partial H}{\partial y}-M\left(u-u_{e}\right) \sin ^{2} \alpha
\end{aligned}
$$

Energy equation:

$u \frac{\partial \theta}{\partial x}+v \frac{\partial \theta}{\partial y}=\frac{1}{\operatorname{Pr}} \frac{\partial^{2} \theta}{\partial y^{2}}$

Micropolar equation:

$$
u \frac{\partial H}{\partial x}+v \frac{\partial H}{\partial y}=-K_{1}\left(2 H+\frac{\partial u}{\partial y}\right)+\left(1+\frac{K_{1}}{2}\right) \frac{\partial^{2} H}{\partial y^{2}}
$$

The boundary conditions in Eq. (5) are transformed to

$$
\begin{aligned}
& u=v=0, \quad \theta=1, \quad H=-n \frac{\partial u}{\partial y} \quad \text { on } \quad y=0, \\
& u=u_{e}(x), \quad \frac{\partial u}{\partial y}=0, \quad \theta=0, \quad H=0 \quad \text { as } \quad y \rightarrow \infty
\end{aligned}
$$

where $\operatorname{Pr}$ is the Prandtl number, $\lambda$ is the constant mixed convection parameter, $K$ represents the dimensionless viscoelastic parameter while $K_{1}$ is the micropolar or material parameter. The parameters are defined as :

$K=\frac{k_{0} U_{\infty}}{a \rho v}, \quad K_{1}=\frac{\kappa}{\mu}, \quad \lambda=\frac{G r}{\operatorname{Re}^{2}}$

and the Grashoff number is $G r=\frac{g \beta\left(T_{w}-T_{\infty}\right) a^{3}}{v^{2}}$.

In order to reduce the complexity of Eq. (7) to (10) according to the boundary conditions (11), the following variables are introduced.

$\psi=x f(x, y), \theta=\theta(x, y), H=x G(x, y)$

where $\psi$ is the stream function defined as,

$u=\frac{\partial \psi}{\partial y}, \quad v=-\frac{\partial \psi}{\partial x}$

By substituting Eq. (12) and (13) into Eq. (8) to (11), new version of the equations are obtained.

$$
\begin{aligned}
& \left(1+K_{1}\right) \frac{\partial^{3} f}{\partial y^{3}}+f \frac{\partial^{2} f}{\partial y^{2}}-\left(\frac{\partial f}{\partial y}\right)^{2}+\frac{\sin x \cos x}{x}+\lambda \frac{\sin x}{x} \theta+K_{1} \frac{\partial G}{\partial y}-M\left(\frac{\partial f}{\partial y}-\frac{\sin x}{x}\right) \sin ^{2} \alpha \\
& +K\left\{2 \frac{\partial f}{\partial y} \frac{\partial^{3} f}{\partial y^{3}}-f \frac{\partial^{4} f}{\partial y^{4}}-\left(\frac{\partial^{2} f}{\partial y^{2}}\right)^{2}+x\left(\begin{array}{l}
\frac{\partial^{2} f}{\partial x \partial y} \frac{\partial^{3} f}{\partial y^{3}}-\frac{\partial f}{\partial x} \frac{\partial^{4} f}{\partial y^{4}} \\
+\frac{\partial f}{\partial y} \frac{\partial^{4} f}{\partial x \partial y^{3}}-\frac{\partial^{2} f}{\partial y^{2}} \frac{\partial^{3} f}{\partial x \partial y^{2}}
\end{array}\right)\right\} \\
& =x\left(\frac{\partial f}{\partial y} \frac{\partial^{2} f}{\partial x \partial y}-\frac{\partial f}{\partial x} \frac{\partial^{2} f}{\partial y^{2}}\right)
\end{aligned}
$$

$$
\begin{aligned}
& \frac{1}{\operatorname{Pr}} \frac{\partial^{2} \theta}{\partial y^{2}}+f \frac{\partial \theta}{\partial y}=x\left(\frac{\partial f}{\partial y} \frac{\partial \theta}{\partial x}-\frac{\partial f}{\partial x} \frac{\partial \theta}{\partial y}\right) \\
& \left(1+\frac{K_{1}}{2}\right) \frac{\partial^{2} G}{\partial y^{2}}+f \frac{\partial G}{\partial y}-\frac{\partial f}{\partial y} G-K_{1}\left(2 G+\frac{\partial^{2} f}{\partial y^{2}}\right)= \\
& x\left(\frac{\partial f}{\partial y} \frac{\partial G}{\partial x}-\frac{\partial f}{\partial x} \frac{\partial G}{\partial y}\right)
\end{aligned}
$$

subject to the boundary conditions $f=\frac{\partial f}{\partial y}=0, \quad \theta=1, \quad G=-\frac{1}{2} \frac{\partial^{2} f}{\partial y^{2}} \quad$ at $\quad y=0$

$\frac{\partial f}{\partial y}=\frac{\sin x}{x}, \quad \frac{\partial^{2} f}{\partial y^{2}}=0, \quad \theta=0, \quad G=0 \quad$ as $\quad y \rightarrow \infty$

From Eq. (17), $n$ is a constant which value lies between and including 0 and 1 . The value $n=0$ indicates strong interaction of microelemets that they are unable to rotate close to the wall surface (Guram and Smith, 1980) while $n=1$ is used to represent turbulent boundary layer flow (Peddieson, 1972). As for the case $n=1 / 2$, which is chosen for this study, the value denotes weak concentration and disappearance of the anti-symmetric part of the stress tensor (Ahmadi, 1976).

At lower stagnation point of the cylinder, i.e. $x \approx 0$ Eq. (14) to (17) will reduce to the following ordinary differential equations:

$$
\begin{aligned}
& \left(1+K_{1}\right) f^{\prime \prime \prime}+f f^{\prime \prime}-f^{\prime 2}+1+\lambda \theta+K\left(2 f^{\prime} f^{\prime \prime \prime}-f f^{i v}-f^{\prime \prime 2}\right) \\
& \quad+K_{1} G^{\prime}-M\left(f^{\prime}-1\right) \sin ^{2} \alpha=0 \\
& \frac{1}{\operatorname{Pr}} \theta^{\prime \prime}+f \theta^{\prime}=0 \\
& \left(1+\frac{K_{1}}{2}\right) G^{\prime \prime}+f G^{\prime}-f^{\prime} G-K_{1}\left(2 G+f^{\prime \prime}\right)=0
\end{aligned}
$$

subject to the boundary conditions

$$
\begin{aligned}
& f(0)=f^{\prime}(0)=0, \quad \theta(0)=1, \quad G(0)=-\frac{1}{2} f^{\prime \prime}(0) \quad \text { at } y=0 \\
& f^{\prime}(\infty)=1, \quad f^{\prime \prime}(\infty)=0, \quad \theta(\infty)=0, \quad G(\infty)=0 \quad \text { as } y \rightarrow \infty
\end{aligned}
$$

where primes denote differentiation with respect to $y$.

Using the finite difference method known as the Keller-box along with the Newton's linearization technique as described by (Cebeci and Bradshaw, 1984), Eq. (14) - (21) were solved numerically using the Fortran programming.

\section{RESULTS AND DISCUSSION}

It is essential to highlight that if the viscoelasticity, aligned MHD and micropolar effects are absent (i.e $K=M=K_{1}=0$ ), the current problem will be transformed to the study conducted by (Merkin, 1977). The previous study focusses on the mixed convection of a heated and cooled circular cylinder. Basically, the current study is an extension on Merkin's model when fluid of interest is viscoleastic micropolar with existance of magnetic effect. Due to the similarity of both studies, for validation purpose the current and prior results are compared. Based on the evident resemblance of the output in Table 1 for values of skin friction coefficient, $f^{\prime \prime}(0)$ and heat transfer, $-\theta^{\prime}(0)$

at numerous values of $\lambda$ and $\operatorname{Pr}=1$, authors are assured that the computation of the proposed model is plausible. The margin of error between Merkin's and current model are too small that it can be ignored. The values of $f^{\prime \prime}(0)$ and $-\theta^{\prime}(0)$ for various values of $K$ when

$M=0.5, K_{l}=0.5, \alpha=\frac{\pi}{4}$ and $\operatorname{Pr}=25$ are shown in Table 2. A large value of Prandtl number is selected to fit the characteristic of being a viscoleastic microplar fluid which is from the non-Newtonian family. It can be observed from the result that as we fixed the other parameters, as $K$ increases, both the values of $f^{\prime \prime}(0)$ and $-\theta^{\prime}(0)$ decrease. It is also evident that across the different $K$ values, $f^{\prime \prime}(0)$ and $-\theta^{\prime}(0)$ increases along with the value of $\lambda$. 
Table 1 Comparative study on $f^{\prime \prime}(0)$ and $-\theta^{\prime}(0)$.

\begin{tabular}{|c|c|c|c|c|}
\hline & \multicolumn{2}{|c|}{ Merkin (1977) } & \multicolumn{2}{c|}{ Present } \\
\hline$\lambda$ & $f^{\prime \prime}(0)$ & $-\theta^{\prime}(0)$ & $f^{\prime \prime}(0)$ & $-\theta^{\prime}(0)$ \\
\hline-1.9 & -0.09987 & 0.38467 & -0.099929 & 0.384652 \\
\hline-1.8 & 0.01950 & 0.40993 & 0.019467 & 0.409934 \\
\hline-1.6 & 0.20923 & 0.44409 & 0.209196 & 0.444091 \\
\hline-1.4 & 0.36982 & 0.46907 & 0.369794 & 0.469064 \\
\hline-1.2 & 0.51460 & 0.48935 & 0.514586 & 0.489351 \\
\hline-1.0 & 0.64886 & 0.50667 & 0.648916 & 0.506689 \\
\hline-0.8 & 0.77554 & 0.52193 & 0.775596 & 0.521961 \\
\hline-0.6 & 0.89627 & 0.53566 & 0.896256 & 0.535678 \\
\hline-0.4 & 1.01219 & 0.54818 & 1.012254 & 0.548210 \\
\hline-0.2 & 1.12410 & 0.55973 & 1.124066 & 0.559742 \\
\hline 0.0 & 1.23259 & 0.57047 & 1.232658 & 0.570492 \\
\hline 0.2 & 1.33810 & 0.58052 & 1.338181 & 0.580548 \\
\hline 0.4 & 1.44100 & 0.58999 & 1.439679 & 0.589864 \\
\hline 0.6 & 1.54158 & 0.59895 & 1.541523 & 0.598964 \\
\hline 0.8 & 1.64007 & 0.60747 & 1.640159 & 0.607493 \\
\hline 1.0 & 1.73666 & 0.61559 & 1.736780 & 0.615613 \\
\hline 1.4 & 1.92482 & 0.63079 & 1.922781 & 0.630629 \\
\hline 1.8 & 2.10711 & 0.64484 & 2.106987 & 0.644849 \\
\hline 2.2 & 2.28432 & 0.65792 & 2.281646 & 0.657725 \\
\hline 3.0 & 2.62587 & 0.68173 & 2.622574 & 0.681504 \\
\hline 5.0 & 3.42296 & 0.73151 & 3.422668 & 0.731512 \\
\hline 8.0 & 4.51480 & 0.79017 & 4.506890 & 0.789785 \\
\hline 10.0 & 5.19484 & 0.82264 & 5.195332 & 0.822686 \\
\hline & & & & \\
\hline
\end{tabular}

Table 2 Value of shear stress coefficient at fixed values of $M=0.5$, $K_{1}=0.5, \quad \alpha=\frac{\pi}{4}$ and $\operatorname{Pr}=25$.

\begin{tabular}{|c|c|c|c|c|c|c|}
\hline \multirow{2}{*}{$\lambda$} & \multicolumn{2}{|c|}{$\mathrm{K}=0.1$} & \multicolumn{2}{c|}{$\mathrm{K}=0.5$} & \multicolumn{2}{c|}{$\mathrm{K}=2$} \\
\cline { 2 - 7 } & $f^{\prime \prime}(0)$ & $-\theta^{\prime}(0)$ & $f^{\prime \prime}(0)$ & $-\theta^{\prime}(0)$ & $f^{\prime \prime}(0)$ & $-\theta^{\prime}(0)$ \\
\hline-1.0 & 0.832912 & 1.674174 & 0.670632 & 1.569531 & 0.433940 & 1.381811 \\
\hline-0.5 & 0.934258 & 1.714407 & 0.762295 & 1.609658 & 0.510568 & 1.422824 \\
\hline 0.0 & 1.031369 & 1.751278 & 0.848993 & 1.645828 & 0.581041 & 1.458493 \\
\hline 0.1 & 1.050345 & 1.758306 & 0.865820 & 1.652663 & 0.594530 & 1.465118 \\
\hline 0.6 & 1.143240 & 1.791929 & 0.947700 & 1.685132 & 0.659425 & 1.496152 \\
\hline 1.2 & 1.250814 & 1.82936 & 1.041592 & 1.720861 & 0.732509 & 1.529575 \\
\hline 1.5 & 1.303193 & 1.847052 & 1.086983 & 1.737604 & 0.767401 & 1.545011 \\
\hline
\end{tabular}

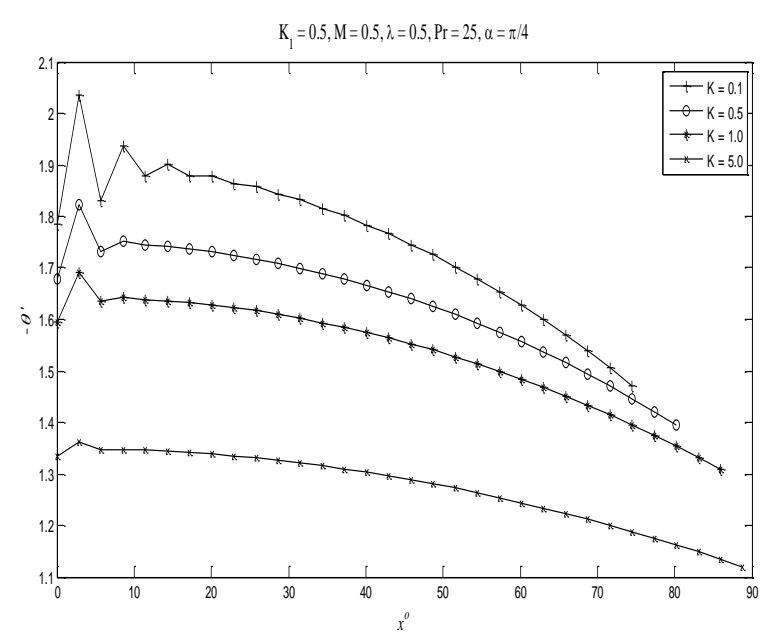

Fig. 2 Heat transfer for various values of $K$.

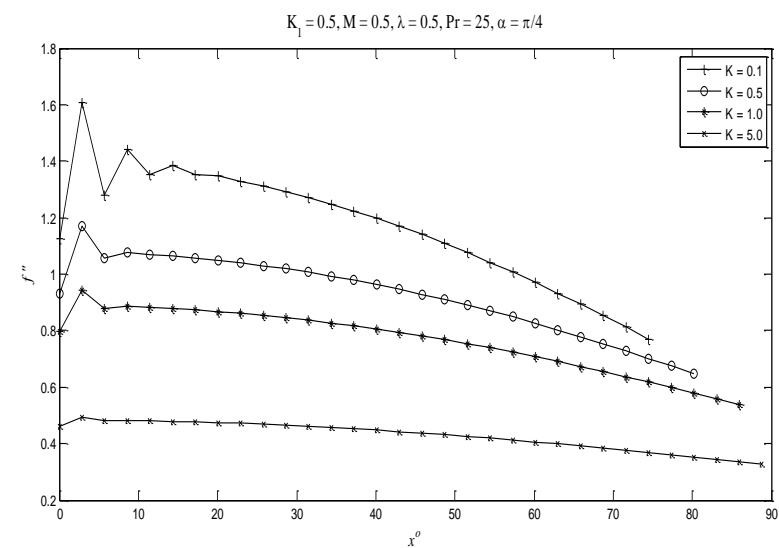

Fig. 3 Skin friction coefficient for various values of $K$.

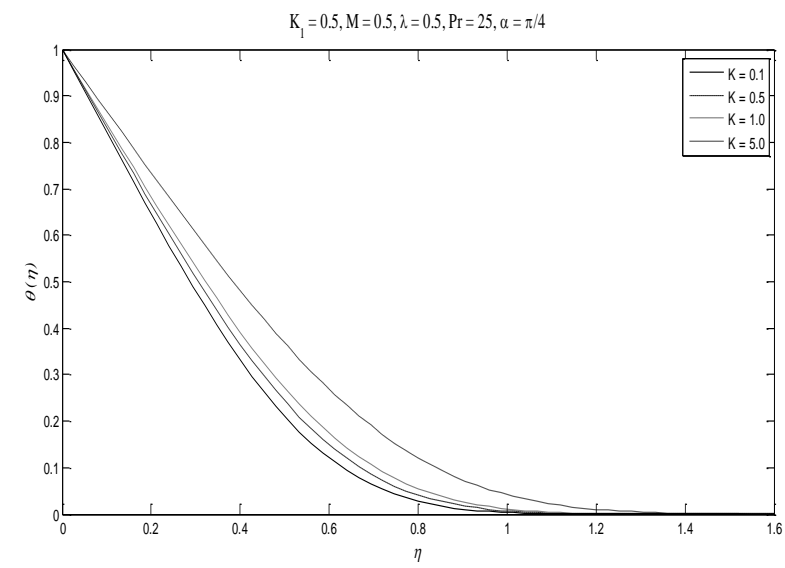

Fig. 4 Temperature distribution of fluid for various values of $K$.

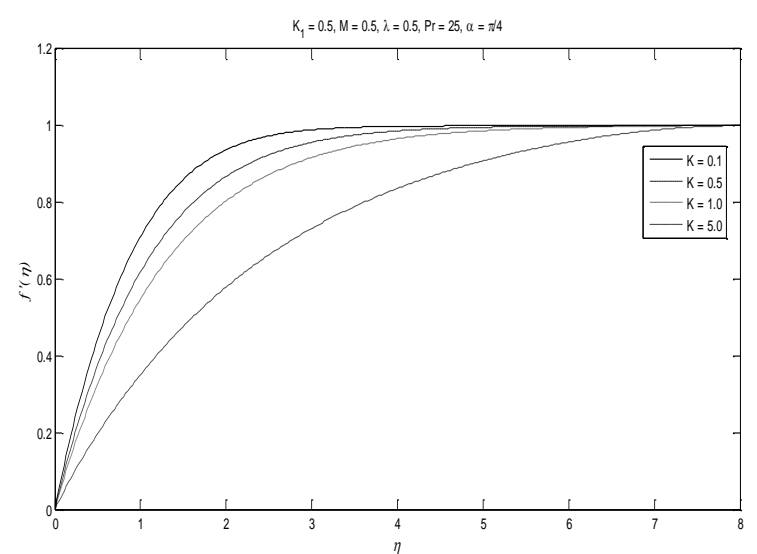

Fig. 5 Velocity distribution of fluid for various values of $K$.

Fig. 2 and 3 shows the variation of the heat transfer and skin friction coefficient distribution with fixed values of $M=0.5, K_{l}=0.5$, $\lambda=0.5, \alpha=\frac{\pi}{4}$ and $\operatorname{Pr}=25$. From the figures, it is apparent that as the viscoleastic parameter grows, the heat transfer and skin friction coefficient decrease. The result turns out as expected since viscoscity is one of the factors that affect the rate of heat transfer in convection as convection currents move faster within fluids with low viscosity than fluids which are highly viscous. It can be observed from the figures that it takes longer for the boundary layer to separate from the cylinder as the value of the viscoelastic parameter, $K$ gets larger. Boundary layer separation is a phenomena that occurs when the boundary layer peels away from a blunt solid surface after being opposed by adverse pressure gradient. This phenomena is undesirable especially in engineering applications as it causes large pressure drag on the cylinder that is even larger than the drag caused by skin friction. It is also noticeable that both figures display peculiar jagged 
lines at the beginning before they get smoother as the flow travels towards the middle sides of the cylinder. This could occur due to the instability of the flow as it first touches the surface of the cylinder at the stagnation point. However, there might also be other causes that authors are not aware of and hence, we welcome ideas from fellow researchers.

The velocity and temperature profiles for variety of $K$ values at the lower stagnation point of the cylinder against the boundary layer thickness, $\eta$ are illustrated in Fig. 4 and 5. The figures prove that fluid viscoelasticity does affect the fluid velocity and temperature profiles. When $K$ increases, the temperature and velocity distributions, increase and decrease, respectively.

\section{CONCLUSION}

The problem of mixed convection boundary layer flow of viscoelastic micropolar fliud with aligned MHD effect is considered in this study. Holding other parameters constant, this study reveals that :

- when the viscoelastic parameter, $K$ gets larger, the heat transfer and skin friction coefficient become smaller.

- the temperature and velocity distributions, increase and decrease, respectively as $K$ increases.

- $\quad$ the boundary layer separates from the cylinder sooner as the value of the viscoelastic parameter, $K$ gets smaller.

\section{ACKNOWLEDGEMENT}

The authors would like to express our gratitude for financial support received from Universiti Malaysia Pahang (UMP) for RDU141306, RDU1703258, RDU161106.

\section{REFERENCES}

Ahmadi, G. (1976). Self-similar solution of imcompressible micropolar boundary layer flow over a semi-infinite plate. International Journal of Engineering Science, 14(7), 639-646.

Alkasasbeh, H. T., Sarif, N. M., Salleh, M. Z., Tahar, R. M., Nazar, R., Pop, I., et al. (2015). Effect of radiation and magnetohydrodynamic free convection boundary layer flow on a solid sphere with Newtonian heating in a micropolar fluid. AIP Conference Proceedings, 1643, 662-669.

Allen, S. J., Kline, K. A. (1971). Lubrication theory for micropolar fluids. Journal of Applied Mechanics, 38(3), 646-650.

Anwar, I., Amin, N., Pop, I. (2008). Mixed convection boundary layer flow of a viscoelastic fluid over a horizontal circular cylinder. International Journal of Non-Linear Mechanics, 43(9), 814-821.

Cebeci, T., Bradshaw, P. (1984). Physical and computational aspects of convective heat transfer. Springer, Berlin Heidelberg.

Dandapat, B. S., and Gupta, A. S. (1989). Flow and heat transfer in a viscoelastic fluid over a stretching sheet. International Journal of NonLinear Mechanics, 24(3), 215-219.

Das, S., Guha, S. K., and Chattopadhyay, A. K. (2004). Theoretical analysis of stability characteristics of hydrodynamic journal bearings lubricated with micropolar fluids. Proceedings of the Institution of Mechanical Engineers, Part J: Journal of Engineering Tribology, 218(1), 45-56.

Ellahi, R., Rahman, S., Gulzar, M. M., Nadeem, S., and Vafai, K. (2014). A mathematical study of non-Newtonian micropolar fluid in arterial blood flow through composite stenosis. Applied Mathematics \& Information Sciences, 8(4), 1567-1573.
Eringen, A. C. (1964). Simple microfluids. International Journal of Engineering Science, 2(2), 205-217.

Eringen, A. C. (1966). Theory of micropolar fluids: West Lafayette, IN: School of Aeronautics and Astronautics, Purdue University.

Eringen, A. C. (2001). Microcontinuum field theories: II. Fluent media New York: Springer.

Guram, G. S., and Smith, A. C. (1980). Stagnation flows of micropolar fluids with strong and weak interactions. Computers \& Mathematics with Applications, 6(2), 213-233.

Huang, P. Y., and Feng, J. (1995). Wall effects on the flow of viscoelastic fluids around a circular cylinder. Journal of Non-Newtonian Fluid Mechanics, 60(2), 179-198.

Ishak, A., Nazar, R., and Pop, I. (2007). Mixed convection stagnation point flow of a micropolar fluid towards a stretching sheet. Meccanica, 43(4), 411.

Jin, H. W., Rajagopalan, S., Ozekcin, A., Haque, T., Ertas, M. D., Zhao, B., et al. (2013). Coated sleeved oil and gas well production devices: Google Patents. US 8286715 B2, https://www.google.com/patents/US8286715

Kasim, A. R. M., Admon, M. A., and Shafie, S. (2011). Free convection boundary layer flow of a viscoelastic fluid in the presence of heat generation. Elastic, 804, 1646.

Mekheimer, K. S., Elnaqeeb, T., El Kot, M., and Alghamdi, F. (2016). Simultaneous effect of magnetic field and metallic nanoparticles on a micropolar fluid through an overlapping stenotic artery: Blood flow model. Physics Essays, 29(2), 272-283.

Merkin, J. (1977). Mixed convection from a horizontal circular cylinder International Journal of Heat and Mass Transfer, 20, 73-77.

Misra, J., Chandra, S., Shit, G., and Kundu, P. (2014). Electroosmotic oscillatory flow of micropolar fluid in microchannels: Application to dynamics of blood flow in microfluidic devices. Applied Mathematics and Mechanics, 35(6), 749-766.

Mohd Kasim, A. R. M. K., Mohammad, N. F. M., Shafie, S., Pop, I. (2013). Constant heat flux solution for mixed convection boundary layer viscolastic fluid. Heat Mass Transfer, 49, 163-171.

Naduvinamani, N. B., Kadadi, A. K. (2013). Effect of Viscosity variation on the micropolar fluid squeeze film lubrication of a short journal bearing. Advances in Tribology, 2013, vol. 2013, Article ID 743987.

Nazar, R., Amin, N., Filip, D., and Pop, I. (2004). Stagnation point flow of a micropolar fluid towards a stretching sheet. International Journal of NonLinear Mechanics, 39(7), 1227-1235.

Nazar, R., Amin, N., and Pop, I. (2003). Mixed convection boundary-layer flow from a horizontal circular cylinder in micropolar fluids: Case of constant wall temperature. International Journal of Numerical Methods for Heat \& Fluid Flow, 13(1), 86-109.

Peddieson, J. (1972). An application of the micropolar fluid model to the calculation of a turbulent shear flow. International Journal of Engineering Science, 10(1), 23-32.

Qasim, M., Khan, I., and Shafie, S. (2013). Heat transfer in a micropolar fluid over a stretching sheet with Newtonian heating. PloS one, 8(4), e59393.

Rahmatabadi, A. D., Nekoeimehr, M., and Rashidi, R. (2010). Micropolar lubricant effects on the performance of noncircular lobed bearings. Tribology International, 43(1-2), 404-413.

Rajagopal, K. R., Na, T. Y., and Gupta, A. S. (1984). Flow of a viscoelastic fluid over a stretching sheet. Rheologica Acta, 23(2), 213-215.

Salleh, M. Z., Nazar, R., Zaharim, A., and Sopian, K. (2009). Free convection boundary layer flow near the lower stagnation point of a sphere with Newtonian heating in a micropolar fluid. Proceedings of the 4th IASME/WSEAS International Conference on Continuum Mechanics '09, WSEAS Press, 174-180.

Ustinov, S. (2016). Features of selection of flow measurement methods and devices for flow measuring of liquefied petroleum gas in pipelines. Undegraduate Thesis, Saimaa University of Applied Sciences Technology, Lappeenranta

Xiong, Y. L., Bruneau, C. H., and Kellay, H. (2010). Drag enhancement and drag reduction in viscoelastic fluid flow around a cylinder. Europhysics Letters, 91, 64001. 